\title{
Laboratory experiment of checkerboard pupil mask coronagraph
}

\author{
K. Enya ${ }^{1}$, S. Tanaka ${ }^{1,2}$, L. Abe ${ }^{3}$, and T. Nakagawa ${ }^{1}$ \\ 1 Department of Infrared Astrophysics, Institute of Space and Astronautical Science, Japan Aerospace Exploration Agency, \\ Yoshinodai 3-1-1, Sagamihara, Kanagawa 229-8510, Japan \\ e-mail: enya@ir.isas.jaxa.jp \\ 2 Department of Physics, Graduate School of Science, University of Tokyo, Hongo 7-3-1, Bunkyo-ku, Tokyo 113-0033, Japan \\ 3 Optical and Infrared Astronomy Division \& Extra-solar Planet Project Office, National Astronomical Observatory, Osawa 2-21-2, \\ Mitaka, Tokyo 181-8588, Japan
}

Received 6 September 2006 / Accepted 20 September 2006

\section{ABSTRACT}

\begin{abstract}
Context. We present the results of the first laboratory experiment of checkerboard shaped pupil binary mask coronagraphs using visible light, in the context of the R\&D activities for future mid-infrared space missions such as the $3.5 \mathrm{~m}$ SPICA telescope.

Aims. The primary aim of this work is to demonstrate the coronagraphic performance of checkerboard masks down to a $10^{-6}$ peakto-peak contrast, which is required to detect self-luminous extra-solar planets in the mid-infrared region.

Methods. Two masks, consisting of aluminum films on a glass substrates, were manufactured using nano-fabrication techniques with electron beam lithography: one mask was optimized for a pupil with a $30 \%$ central obstruction and the other was for a pupil without obstruction. The theoretical contrast for both masks was $10^{-7}$ and no adaptive optics system was employed.

Results. For both masks, the observed point spread functions were quite consistent with the theoretical ones. The average contrast measured within the dark regions was $2.7 \times 10^{-7}$ and $1.1 \times 10^{-7}$.

Conclusions. The coronagraphic performance significantly outperformed the $10^{-6}$ requirement and almost reached the theoretical limit determined by the mask designs. We discuss the potential application of checkerboard masks for mid-infrared coronagraphy, and conclude that binary masks are promising for future high-contrast space telescopes.
\end{abstract}

Key words. instrumentation: high angular resolution - methods: laboratory - techniques: miscellaneous

\section{Introduction}

Direct detection and spectroscopy of extra-solar planets is very important to the understanding of how planetary systems were born, how they evolve, and ultimately to find biological signatures on these planets. After the first report unambiguously confirming the existence of an extrasolar planet by Mayor \& Queloz (1995), more than 200 extra-solar planets have been found to date, mostly by detailed observation of the Doppler shift of the spectrum of the central star (Mayor \& Queloz 1995), or by the variability of the luminosity of the central star due to the transit of the planet (Henry et al. 2000; Charbonneau et al. 2000). However, such indirect observation does not usually provide the spectral features, luminosity and other important properties of the planets themselves, so the direct observation of extra-solar planets is of primary importance. However, the enormous contrast in luminosity between the central star and the planet, and the associated diffraction patterns from optical instruments requires some coronagraphic techniques to suppress, or at least attenuate them. Typically, the contrast at visible light wavelengths is $\sim 10^{10}$ but is reduced to $\sim 10^{6}$ in the mid-infrared region (Burrows et al. 2004), which is one of the most important advantages of infrared coronagraphy for the observation of extra-solar planets.

The Space Infrared telescope for Cosmology and Astrophysics (SPICA) is the next generation mission for infrared astronomy led by Japan (Nakagawa et al. 2004). The SPICA telescope uses on-axis Ritchey-Chretien optics with a $3.5 \mathrm{~m}$ diameter monolithic primary mirror. The whole telescope will be cooled to $4.5 \mathrm{~K}$ and infrared observations will be made at wavelengths within the $5-200 \mu \mathrm{m}$ range. A coronagraphic instrument is currently being considered for the SPICA mission (Enya et al. 2006; Abe et al. 2006). The primary target of this instrument is the direct detection and observation of extra-solar Jovian outer planets (typically beyond $5 \mathrm{AUs}$ ). The baseline requirements for the SPICA coronagraph are as follows: $10^{-6}$ peak-to-peak contrast, smallest possible inner-working-angle (IWA), and a core wavelength within the range 5-20 $\mu \mathrm{m}$.

Of the various current coronagraphic methods, coronagraphs using binary shaped pupil masks have some remarkable advantages (e.g., Jacquinot \& Roizen-Dossier 1969; Vanderbei et al. 2003; Kasdin et al. 2003). They are relatively simple in their implementation, essentially achromatic systems, and robust against pointing errors of the telescope (Green et al. 2004). Therefore, binary masks have been studied as the baseline for the SPICA coronagraph development (Tanaka et al. 2006; Enya et al. 2006). Our aim is to demonstrate precise mask fabrication, and to check the performance of the masks with visible light in the laboratory before proceeding further.

In this paper, we present the results of a laboratory demonstration of the checkerboard mask coronagraphs in visible light. The target contrast to be demonstrated in this work was set to $10^{-6}$, which is required to detect self-luminous extra-solar planets in the mid-infrared. It was shown that the observed contrast went beyond this goal, very close to the theoretical contrast of $10^{-7}$. Expansion toward a mid-infrared demonstration test-bench is also discussed. 
Table 1. Design of the two binary checkerboard mask.

\begin{tabular}{lcc}
\hline \hline & Mask 1 & Mask 2 \\
\hline Type & Symmetric & Symmetric \\
Central obstruction & $30 \%$ & No obstruction \\
IWA $^{*}(\lambda / D)$ & 7 & 3 \\
OWA $^{*}(\lambda / D)$ & 16 & 30 \\
Contrast & $10^{-7}$ & $10^{-7}$ \\
Throughput** $(\%)$ & 16 & 24 \\
\hline
\end{tabular}

${ }^{*}$ In the experiment the wavelength was $\lambda=632.8 \mathrm{~nm}$, and the diameter of the entrance pupil was $D=2 \mathrm{~mm}$ (the binary mask is inscribed to this pupil). ${ }^{* *}$ Ratio of the transmissive area of the mask to the whole area for an assumed entrance pupil diameter of $2 \mathrm{~mm}$.

\section{Experiment}

\subsection{Pupil mask}

Recently, Tanaka et al. (2006) has studied the performance of binary masks (circular and checkerboard types) shaped for a pupil comprising a central obstruction and spiders, and an asymmetric checkerboard mask design was presented. For this work, we selected symmetric checkerboard masks because they can be also optimized for the expected SPICA telescope pupil geometry. Moreover, checkerboard masks consist of rectangular patterns which are suitable for the fabrication process used. The reason that asymmetric checkerboard masks were not chosen was only in order to make the fabrication simple; in fact, the fabrication of the asymmetric masks would basically present no additional manufacturing problems.

Optimization of the mask shape was performed with the LOQO solver presented by Vanderbei (1999) to find the solution which maximizes the pupil throughput under the constraints of a given contrast, IWA and outer working angle (OWA). The optimization for checkerboard masks is simply computed in 1D which provides the 2D so-called "bar-code" mask solution. The final 2D mask is simply the combination of two of these masks, rotated by 90 degrees with respect to each other. Therefore, the useful IWA for the 2D masks is $\sqrt{2}$ times larger than the IWA defined for the optimized bar-code mask. Note that in this paper, all measurements are made in diagonally from the center of the image in order to match the correct IWA definition.

In the optimized solutions providing throughput higher than $15 \%$, the design which had the smallest IWA was adopted for manufacture. The targeted contrast to be demonstrated in this work was $10^{-6}$, so that the mask was designed to achieve a contrast of $10^{-7}$. The size chosen for the central obstruction of the first mask (Mask 1, IWA of $7 \lambda / D$ ) was $30 \%$ of the entrance pupil diameter. In our experiment, the wavelength was $\lambda=632.8 \mathrm{~nm}$ and the diameter of the entrance pupil $D=2 \mathrm{~mm}$ (i.e. the diagonal of our square masks). Subsequently, for comparison, a second mask (Mask 2) was designed for a pupil without obstruction, and to probe the coronagraphic sensitivity at much lower IWA $(3 \lambda / D)$. The OWAs for Masks 1 and 2 were assumed to be 16 and $30 \lambda / D$. The smaller OWA gave a solution where the rectangular patterns of the mask were not too narrow and therefore made the fabrication simple and robust. The larger OWA provided a wider dark region while it made the fabrication more complex. Table 1 summarizes the specifications of the masks. Figure 1 shows the shape of the masks, and the expected coronagraphic point spread function (PSF) for Mask 1 and Mask 2.

Each checkerboard mask consists of an aluminum film on a BK7 substrate, and were manufactured using nano-fabrication technology at the National Institute of Advanced Industrial

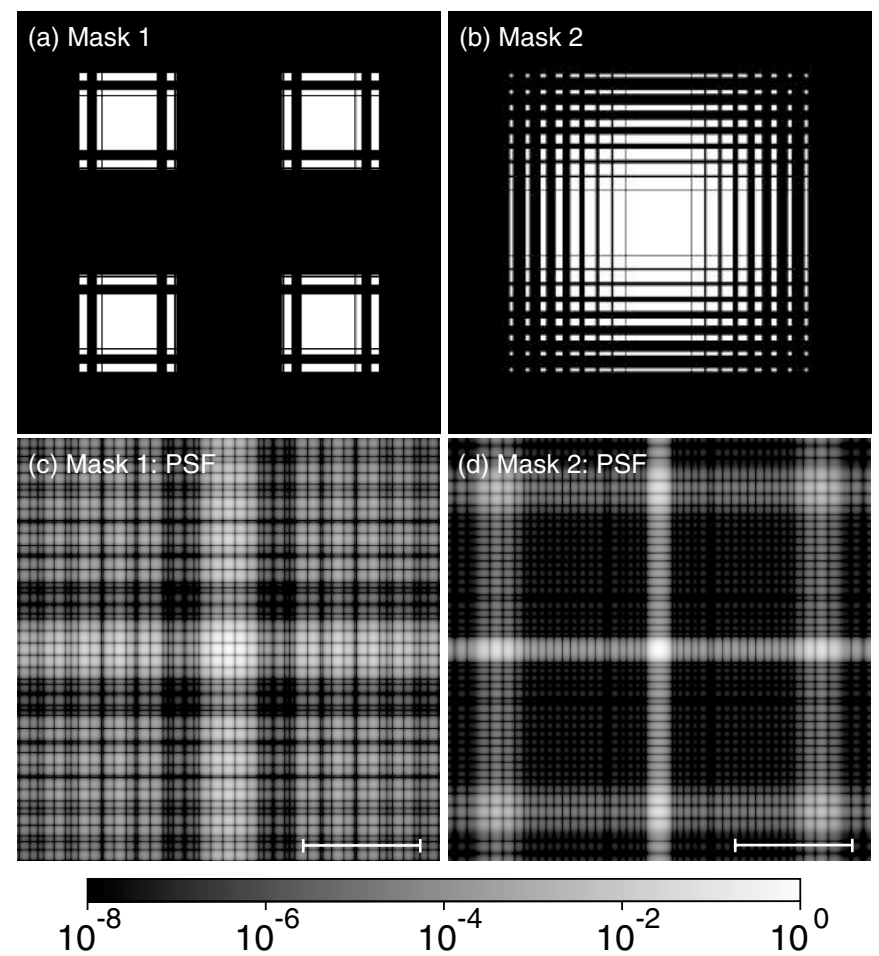

Fig. 1. Panels a) and b) show the Mask 1 and Mask 2 designs. The transmission through the black and white regions is 0 and 1 , respectively. The diameter of the circumscribed circle to the transmissive part is $2 \mathrm{~mm}$. Panels c) and d) show the expected (theoretical) PSFs for Masks 1 and 2. The respective diagonal profiles are shown in Fig. 5. The scale bar is $20 \lambda / D$.

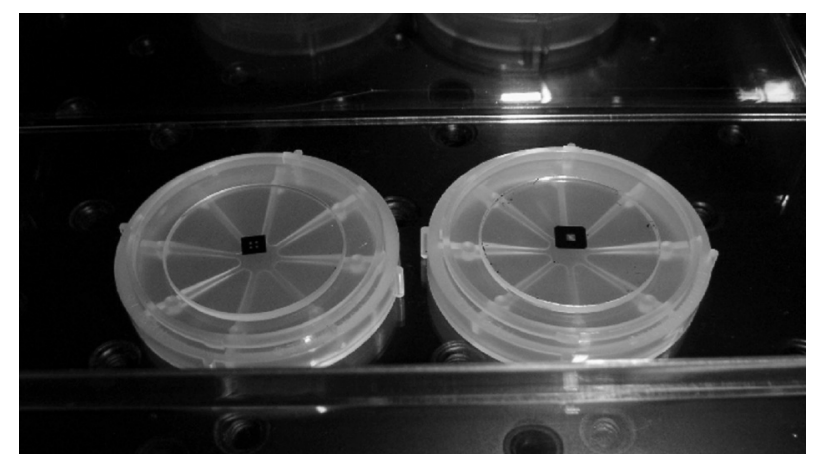

Fig. 2. Photographs showing the fabricated Mask 1 (left) and Mask 2 (right) on BK 7 substrates. The diameter and thickness of the substrates is $30.0 \mathrm{~mm}$ and $2.0 \mathrm{~mm}$, respectively. The translucent cases beneath the substrates are used to transport the devices.

Science and Technology (AIST) in Japan. A $100 \mathrm{~nm}$ thick film aluminum was evaporated onto the surface of the substrate. After evaporation, electron beam patterning and a lift-off process were applied to produce a rectangular transmissive area for the checkerboard pattern. The substrate was $2 \mathrm{~mm}$ thick and had no wedge angle. The flatness, parallelism and scratch-and-dig of the substrate were less than $\lambda / 10,5$ arcsecond and $10^{-5}$ respectively. A narrow-band multiple-layer coating was applied to both sides of the substrate to reduce reflection at the surface after fabrication. The temperature of the mask during coating was kept less than $100{ }^{\circ} \mathrm{C}$ to avoid deterioration of the residual resist around the mask pattern. The reduction in reflectivity of the BK7 substrate was confirmed to be less than $0.25 \%$ as a result of this coating. Figure 2 shows photographs of the final masks 


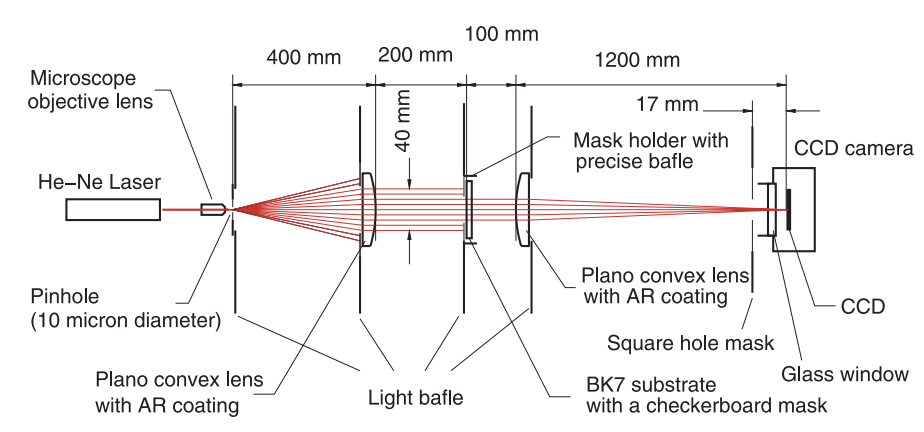

Fig. 3. Optical configuration for the experiment. All the optical devices were set on a table with air suspension in a dark room with an air cleaning system flowing from the top of the room onto the optical table. The air flow was either turned on or off to check its influence on the coronagraphic performance (see text for details).

on their substrates. Small defects were found on Mask 2, but we found no significant degradation in performance for this level of contrast (Sect. 3).

\subsection{Optical setup}

The configuration of the experiment is shown in Fig. 3. All the optics were set in a dark room with an air cleaning system. Clean air flowed from the top of the room to the optical table during the settings and measurements. Although we plan to use an adaptive optics system in future experiments, it was not used in this work. A He-Ne laser was used as the light source. A spatial filter consisting of a microscope objective lens and a $10 \mu \mathrm{m}$ diameter pinhole was also used.

A $50 \mathrm{~mm}$ diameter BK7 plano-convex lens was used for collimating the entrance beam. The lens is a commercially available one, and the surface quality was better than $\lambda / 2$ (over a $30 \mathrm{~mm}$ diameter). The quality of the collimation was checked and adjusted by a Hartman test. The error in the residual wavefront caused by alignment errors in the collimation lens was in $\pm \lambda / 600$. A usual narrow-band multiple-layer anti-reflection coating was applied to both sides of the lens to reduce reflection at the surface. The residual reflectivity was confirmed to be less than $0.25 \%$. A light baffle with an edge thickness of $50 \mu \mathrm{m}$ was used to produce a collimated beam of $40 \mathrm{~mm}$ diameter.

The pupil mask was fixed in a holder with a precisely manufactured baffle with a circular hole made by electrical discharge machining. The thickness of this baffle was $20 \mu \mathrm{m}$ and the diameter of the hole was $3.5 \mathrm{~mm}$. A $20 \mu \mathrm{m}$ thick spacer was used between this mask and the pupil mask. A black coating was applied to both sides of the baffle and the spacer.

Another plano-convex lens was used for focusing. The focal length of this lens was $1200 \mathrm{~mm}$ and the other specifications were the same as those of the collimating lens.

A commercially available cooled CCD camera (BITRAN) with $2048 \times 2048$ pixels was used to measure the PSF. The overall chip size was $15.16 \mathrm{~mm} \times 15.16 \mathrm{~mm}$ with a flat glass window in front. The CCD was cooled and stabilized at $0{ }^{\circ} \mathrm{C}$ throughout the experiment. The camera was mounted on a linear motor drive stage in order to scan along the optical axis to find the best focus position.

If the mask is well aligned to the optical axis, reflected light (due to reflection from the mask itself) produces a very well focused point on, or very close to, the pinhole substrate, which is generally reflective. To prevent such reflection, whole of the pupil mask holder with the mask and the thin baffle was tilted
0.5 degree from the optical axis in the direction of the diagonal of the mask. This ensured that the backward focused spot was far away from any potentially reflective surface, and thus deviated sufficiently from the optical axis. The CCD camera was also tilted by 0.5 degree but in the opposite direction to the mask in order to avoid multiple reflections between these two components. Other devices were set perpendicular to the optical axis. The angular accuracy of the setup was $\sim 0.1$ degree and the accuracy of the centering was $\sim 0.2 \mathrm{~mm}$.

\subsection{Measurement}

The dynamic range of the CCD was insufficient to capture the full dynamic range of the PSF in one single exposure. Therefore, several exposure times were used: $0.03,0.1,1.0,10,100$, and $1800 \mathrm{~s}$. The $1800 \mathrm{~s}$ exposure was for measurements in the dark region. The brightest region of the PSF saturated the detector even with the shortest exposure time, so we used additional neutral density filters with an optical density of 2 . We also carefully checked the CCD linearity by imaging the PSF core through a combination of neutral density filters with a total optical density of 6 , and with a $1800 \mathrm{~s}$ exposure. We found that no significant correction was needed so that we could rely on the multiexposure time method.

For measurements of the dark region, we used a squareshaped mask located just in front of the camera window to block the flux outside the dark region. Without this, the contrast did not exceed $10^{-5}$ because of inner scattering or multi-reflections within the camera surfaces. The role of this mask is equivalent to the "bow tie mask" shown in Belikov et al. (2006), Kasdin et al. (2005), though our mask was set farther in front of the camera window $(\sim 17 \mathrm{~mm})$. The size of the square aperture was $2.3 \mathrm{~mm} \times 2.3 \mathrm{~mm}$ for pupil Mask 1 and $8.0 \mathrm{~mm} \times 8.0 \mathrm{~mm}$ for pupil Mask 2 with the corners of the aperture having a radius of $0.2 \mathrm{~mm}$. The thickness of the mask was $20 \mu \mathrm{m}$ and a black coating was applied to both sides. The mask was set so that the corners of the aperture were $7.5 \lambda / D$ and $3.5 \lambda / D$ distant from the core of the PSF for the experiments with Mask 1 and Mask 2, respectively.

After every exposure for the PSF measurement, we blocked the beam with a black plate placed close to the CCD side of the pupil mask, and took a dark image with the same exposure time. Therefore, this image includes the influence of both the effect of the dark current of the CCD and the background light. This dark frame was subtracted from the corresponding PSF image.

\section{Result and discussion}

Observed coronagraphic images and their profiles with Masks 1 and Masks 2 are shown in Figs. 4 and 5. For both masks, the profiles of the core of the PSF are quite consistent with those expected from theory. The majority of the area of the dark region in the PSF with Mask 1 is less than $10^{-6}$ as shown in Fig. 5. On average, the observed contrast for each dark region on a linear scale is $3.4 \times 10^{-7}, 2.1 \times 10^{-7}, 1.8 \times 10^{-7}$ and $3.5 \times 10^{-7}$ for DR 1 , DR 2, DR 3 and DR 4 respectively, where DR $1 \sim$ DR 4 are the dark regions corresponding to the quadrants around the core shown in Fig. 4. The average contrast for all the dark regions is $2.7 \times 10^{-7}$. The $3 \sigma$ detection limit computed within each observed dark region DR $1 \sim$ DR 4 is $7.5 \times 10^{-7}, 5.8 \times 10^{-7}, 4.1 \times 10^{-7}$, and $9.1 \times 10^{-7}$, respectively. PSF subtraction or fitting has the potential to provide better performance, though such methods require assumptions about the repeatability of the pattern observed in 


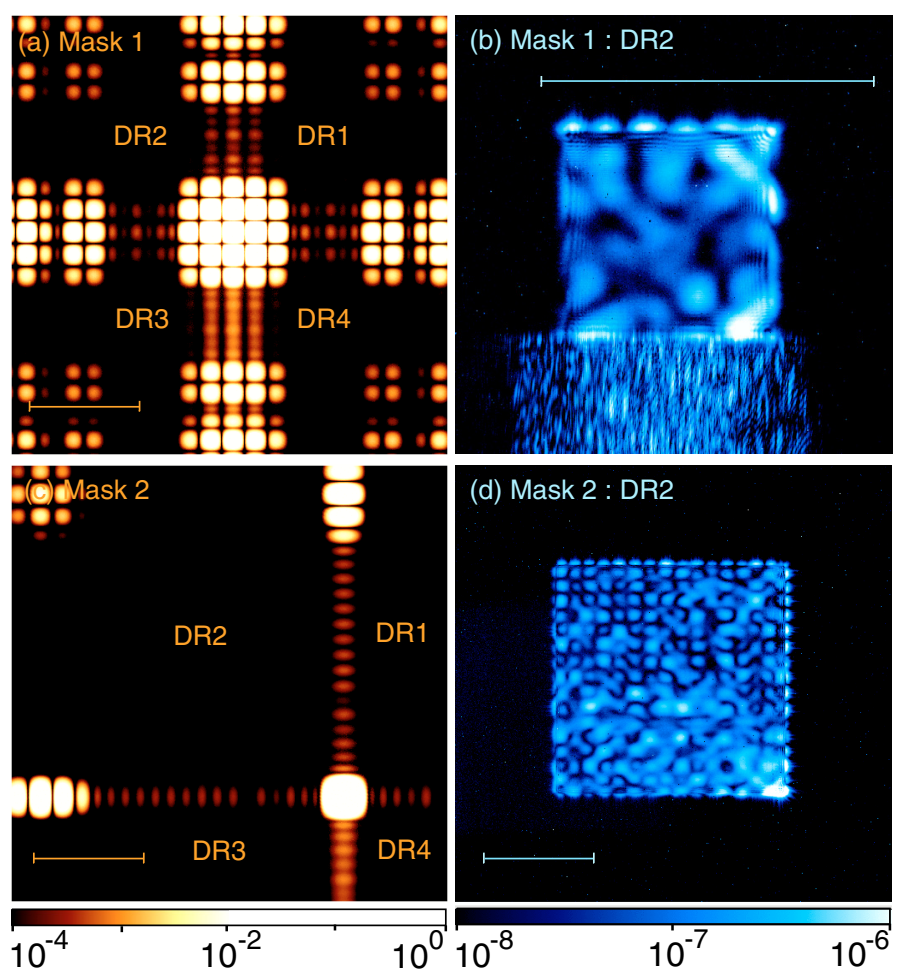

Fig. 4. Observed coronagraphic images. Panels a) and c) show images including the core of the PSF for Masks 1 and 2. The tail below the bright peak is due to a readout effect. Panels b) and d) are images of the dark region obtained with a mask with a square aperture. The prickle like pattern below the square aperture in b) is the result of reflection by the support structure of the aperture for Mask 1 . The scale bar is $10 \lambda / D$.

the dark regions or the shape of the PSF. Therefore, the simple $3 \sigma$ limit corresponds to a conservative estimate of the detection limit. Nevertheless, all the values are below our $10^{-6}$ limit. Considering the effective IWA (including local bright speckles contributions), we can define for each quadrant the distance beyond which the contrast falls below $10^{-6}$. If we call this distance $I W A_{6}$, it is less than $7.5 \lambda / D$ for DR $1, \mathrm{DR} 2$ and DR 3 , while it is $9.5 \lambda / D$ for DR 4 . These results suggest that the average of $I W A_{6}$ is smaller than $8.0 \lambda / D$ and is quite close to theoretical value of $7.0 \lambda / D$.

On the other hand, Fig. 4b exhibits an irregular flux distribution in the dark region, which is not predicted by the theoretical PSF of the mask. Consequently the obtained contrast does not reach the theoretical $10^{-7}$ value. It was confirmed that intensity distribution of the irregular pattern was repeatable in a fixed setup configuration. We did the same measurement with and without air flow or air suspension of the optical table to test the influence of air turbulence or vibration of the devices, and confirmed no significant change occurred. On the contrary, rotating or shifting the mask parallel to its surface changed the shape and intensity of the irregular pattern. Hence, we concluded that errors in the mask fabrication do not dominate the coronagraphic performance of Mask 1. Brighter speckles are caused by a combination of effects in the beam-line: wavefront errors, multi-reflections and scattering by microscopic defects on the surface of the optics and can therefore be reasonably considered as being limiting factors in this experiment.

For the coronagraphic image with Mask 2, the average contrast of DR 1, DR 2, DR 3 and DR 4 on a linear scale is $1.1 \times 10^{-7}$, $1.0 \times 10^{-7}, 9.0 \times 10^{-8}$ and $1.3 \times 10^{-7}$, respectively. The average
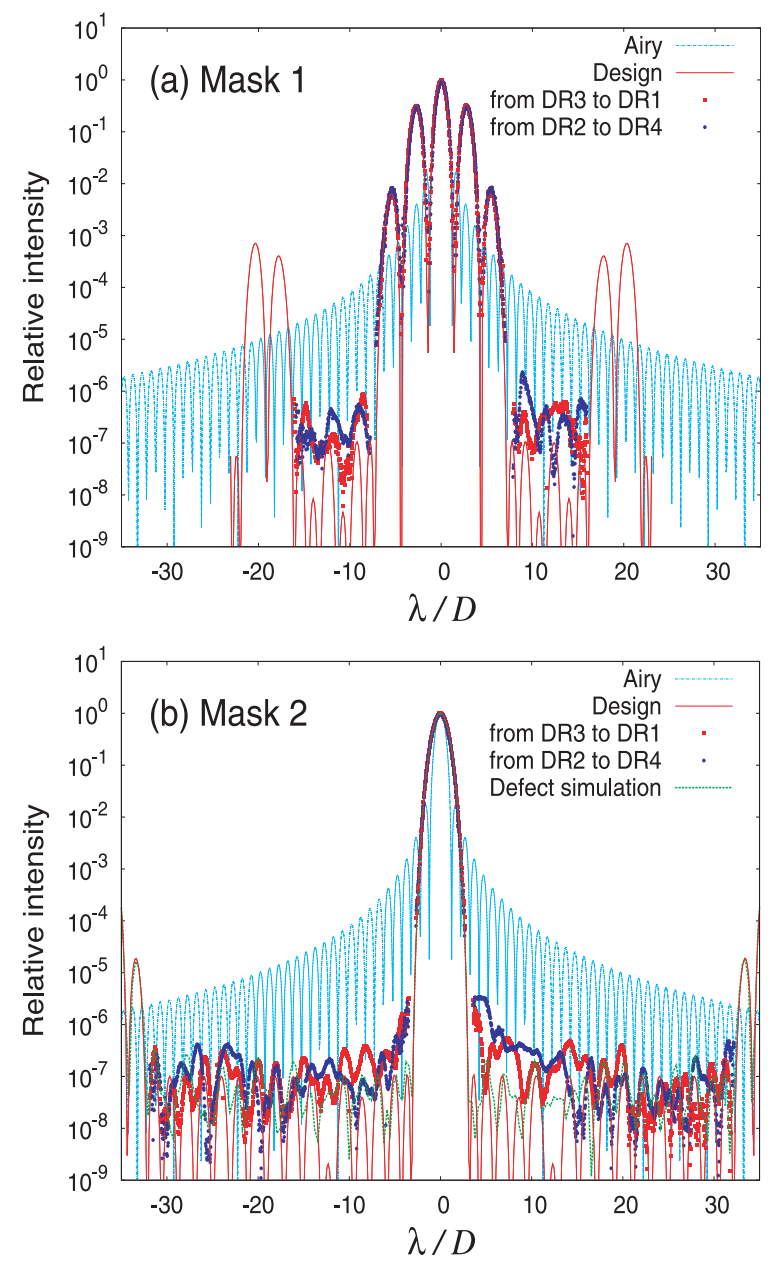

Fig. 5. Diagonal profiles of the coronagraphic image for Mask 1 a) and Mask 2 b). Observed and theoretical profiles are shown as well as the theoretical Airy profile. Each profile is normalized by the peak intensity in each image. For Mask 2, the result of simulation with the observed mask defects shown in Fig. 6 is also presented.

contrast of the whole dark region is $1.1 \times 10^{-7} . I W A_{6}$ for DR $1 \sim$ DR 4 is $4.2 \lambda / D, 4.1 \lambda / D, 4.5 \lambda / D, 5.5 \lambda / D$, respectively. The average of $I W A_{6}$ is $4.6 \lambda / D$. The $3 \sigma$ limit is $3.8 \times 10^{-7}, 3.0 \times 10^{-7}$, $2.7 \times 10^{-7}$ and $3.8 \times 10^{-7}$ for DR 1 , DR 2 , DR 3 and DR 4 respectively. In the bottom part of Fig. 4d, an irregular speckle pattern can be observed, getting brighter close to the optical axis (bottom right). This pattern was invariant in repeated measurements with a fixed setup. Change in the air flow and suspension conditions made no significant difference in the observed image. On the contrary, when we shifted and rotated the mask, this speckle pattern changed, suggesting the same limiting factors as for Mask 1.

On the other hand, a lattice-like pattern was also found at a relatively large distance from the core in the dark region with Mask 2. The position of each lattice "node" agrees quite well with the ones expected from the theoretical image as shown in Fig. 2, but their intensity is not uniform and slightly brighter than that predicted by theory. However, we noticed that their intensity changed while shifting and/or rotating the mask relative to the beam line optics. We therefore concluded that we were observing the theoretical pattern modulated by speckles originating from aberrations in the optics.

We attempted to simulate the effects of the observed defects, in order to check if the recorded intensity of the slightly brighter 

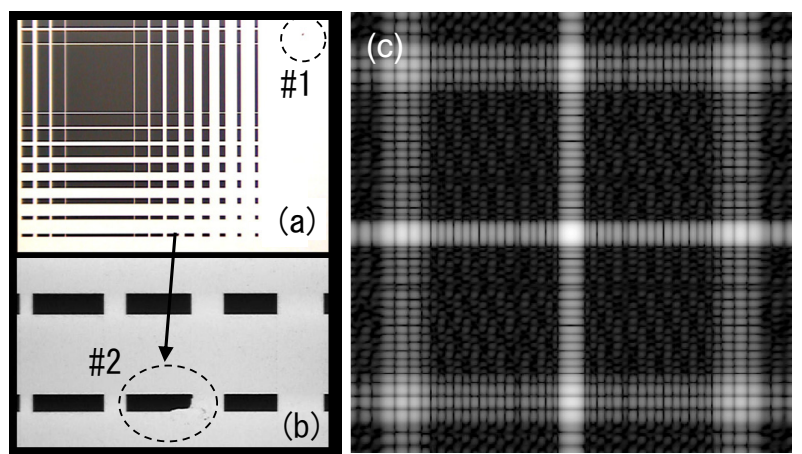

Fig. 6. Panels a) and b) show the defects in Mask 2 observed through an optical microscope using reflected light. The darker parts in the images correspond to the transmitted areas. Panel c) Simulated coronagraphic image including the influence of the observed defects. The color scale is the same as in Fig. 1.

(than theory) lattice pattern was due to these defects. As shown in Figs. $6 a$ and b, two defects were found in Mask 2 by careful inspection of microscopic images. The simulated image including these defects is shown in Fig. 6c, and the corresponding diagonal cut appears in Fig. 5b. To reproduce the observed intensity level of the lattice nodes, a slight overestimate of the size of the defects was needed in the simulation. As a result, we obviously failed to reproduce the observed image because the lattice pattern seen in the top part of Fig. $4 \mathrm{~d}$ is much more similar to the theoretical pattern than to the simulated image with defects. We can therefore confidently conclude that these defects do not significantly degrade the coronagraphic performance at this level of contrast.

Belikov et al.(2006), Kasdin et al.(2005) presented some profiles of coronagraphic PSF using a free standing shaped pupil mask. The performance achieved by our Mask 2 is comparable, or somewhat higher than the previously mentioned results. But the direct comparison must be balanced because the aim and strategy were different. In our experiment, the beam size was quite smaller $(\sim 1 / 10)$, which is definitely an advantage to reduce the wave front error caused by the surface figure of optical components. On the other hand, the centimeter-size beam from, e.g. Belikov et al. (2006) was suitable for using a deformable mirror where the aim was also to test some focal plane speckle attenuation. On-substrate, transmissive components we used can be regarded as being disadvantageous because of the potential multi-reflections and diffusion effects.

As shown above, it has been demonstrated that both checkerboard masks, with or without a large obstruction in the pupil work well, and the observed performance exceeds the target contrast of $10^{-6}$. In principle, it is possible to construct a coronagraph with self standing checkerboard mask and mirrors. Such system is essentially free from the imperfectness of transmissive devices, which provide difficulties of development. The simplicity of the optics is another advantage of the shaped pupil mask coronagraph especially for a general purpose telescope which carries other instruments and therefore the space and weight assigned for the coronagraphic instrument is strictly limited. These facts suggest that a coronagraph using a checkerboard mask is a very promising solution for general purpose infrared telescopes with a significantly obstructed pupil, such as SPICA. On the other hand, beam blocking by the pupil mask, reflected flux from the CCD itself, multi-reflections between the optical devices and inner scattering in the camera can decrease the contrast. Band pass filters or other optical devices required for astronomical purposes are indispensable for the actual instrument, but they make the design of the instrument more complex. Demonstration of the coronagraphic part is therefore not the only critical part to address, and global optimization of all the other components is mandatory in order to achieve the expected performance.

\section{Conclusion}

We report the results from a laboratory experiment using a binary checkerboard-type pupil shaped mask coronagraph. Although this kind of mask presents several limitations (large IWA and low throughput), its manufacture and implementation are very robust. Two masks, optimized for either obstructed pupil or not, were fabricated and tested. Both masks achieved a higher performance than the $10^{-6}$ requirement: $2.3 \times 10^{-7}$ and $1.1 \times 10^{-7}$. Therefore, we conclude that a binary checkerboard mask is a very attractive solution for the future MIR coronagraph of the SPICA space telescope, for which this study was carried out. Other coronagraphic solutions, more complex to implement are also considered, but the checkerboard mask can be regarded as the safest option.

Acknowledgements. We are grateful to T. Wakayama, T. Sato, N. Nakagiri and other colleagues in AIST for their great support in the mask fabrication. We would like to thank all persons relating to SPICA and the SPICA coronagraph. This work was supported in part by a grant from the Japan Science and Technology Agency. L.A. is supported by Grants-in-Aid (No. 160871018002) from the Ministry of Education, Culture, Sports, Science, and Technology (MEXT) of Japan.

\section{References}

Abe, L., Tamura, M., Nakagawa, T., et al. 2005, Proc. IAU Colloq., 200, 329 Belikov, R., Give'on, A., Trauger, J. T., et al. 2006, Proc. SPIE, 6265, in press Burrows, A., Sudarsky, D., \& Hubeny, I. 2004, ApJ, 609, 407

Charbonneau, D., Brown, T. M., Latham, D. W., \& Mayor, M. 2000, ApJ, 529, 45

Enya, K., Shinichiro, T., Nakagawa, T., et al. 2006, Proc. SPIE, 6265, in press Green, J. J., Shaklan, S. B., Vanderbei, R. J., \& Kasdin, N. J. 2004, Proc. SPIE, 5487, 1358

Henry, G. W., Marcy, G. W., Butler, R. P., \& Vogt, S. S. 2000, ApJ, 529, L41

Jacquinot, P., \& Roizen-Dossier, B. 1964, Prog. Opt., 3, 29

Kasdin, N. J., Belikov, R., Beall, J., et al. 2005, Proc. SPIE, 5905, 128

Kasdin, N. J., Vanderbei, R., J., Spergel, D. N., Littman, \& M. G. 2003, ApJ, 582,1147

Mayor, M., \& Queloz, D. 1995, Nature, 378, 355

Nakagawa, T., SPICA working group 2004, Adv. Sp. Res., 34, 645

Spergel, D. N. 2001 [arXiv: asro-ph/0101141]

Tanaka, S., Enya, K., Abe, L., Nakagawa, T., \& Kataza, H. 2006, PASJ, 58, 627

Vanderbei, R. J. 1999, Optimization methods \& software, 21, 485

Vanderbei, R. J., Spergel, D. N., \& Kasdin, N. J. 2003, ApJ, 590, 593

Vanderbei, R. J., Kasdin, N. J., \& Spergel, D. N. 2004, ApJ, 615, 555 